\title{
Acute Hematogenous Infection of Revision Total Hip Arthroplasty by Oral Bacteria in a Patient without a History of Dental Procedures: Case Report
}

\author{
Motoki Sonohata*, Masaru Kitajima, Syunsuke Kawano and Masaaki Mawatari \\ Department of Orthopedic Surgery, Faculty of Medicine, Saga University, 5-1-1 Nabeshima, Saga 849-8501, Japan
}

\begin{abstract}
The risk of periprosthetic joint infection from hematogenous bacterial seeding is increased in patients undergoing dental procedures that facilitate the development of bacteremia.

We herein report the case of a patient without a history of dental procedures who suffered from an acute metastatic infection of a hip prosthesis by the oral bacterium Streptococcus mutans 18 months after undergoing revision total hip arthroplasty. The patient was successfully treated by two-stage revision surgery.

It is important to realize that the efficacy of antibiotic prophylaxis against joint infections has not yet been convincingly proven. As a result, optimal dental hygiene and regular dental visits may be more important than antibiotic prophylaxis for maintaining joint health. Therefore, orthopedic surgeons should educate patients with joint prostheses about good oral health.
\end{abstract}

Keywords: Antibiotic prophylaxis, bacteremia, hematogenous, infection, oral bacteria, Streptococcus mutans, total hip arthroplasty.

\section{INTRODUCTION}

Periprosthetic joint infection is the most common and severe complication following joint replacement. There are many prophylactic methods for decreasing the incidence of surgical site infection; however, late infection due to bacteremia remains difficult to diagnose and treat.

The risk of periprosthetic joint infection from hematogenous bacterial seeding is increased in patients undergoing dental procedures that facilitate the development of bacteremia [1-3]. Although it was previously recommended that patients with major joint prostheses receive antibiotic prophylaxis appropriate to the flora of the oral cavity when dental treatment is undertaken $[4,5]$, this practice is controversial $[6,7]$. The incidence of infection in site of joint replacements due to hematogenous seeding from dental procedures has been reported to be $0.04-0.1 \%$ in reviews [7], and there are many case reports concerning such patients [8-10].

However, there are few reports regarding metastatic infections of site of joint replacement by oral bacteriain patients without a history of dental procedures. We here in report the case of a patient without a history of dental procedures who suffered from an acute metastatic infection of a hip prosthesis by the oral bacteria Streptococcus mutans, 18 months after undergoing revision total hip arthroplasty (THA).

The study protocol adhered to the ethics guidelines of the 1975 Declaration of Helsinki, and the study was approved by

*Address correspondence to this author at the Department of Orthopaedic Surgery, Faculty of Medicine, Saga University, Nabeshima 5-1-1, Saga 849-8501, Japan; Tel: +81-952-34-2343; Fax: +81-952-34-2059;

E-mail: sonohata@cc.saga-u.ac.jp the institutional review board of the Faculty of Medicine, Saga University. The patient was informed that this case study would be submitted for publication, and she provided her informed consent.

\section{CASE}

A 53-year old female underwent cup and ball revision of a right THA due to loosening and osteolysis 17 years after primary surgery using a cementless THA (Fig. 1). She did not take any corticosteroids and had no history of disease associated with a possibility of infection, such as diabetic mellitus, rheumatoid arthritis, liver cirrhosis, etc.

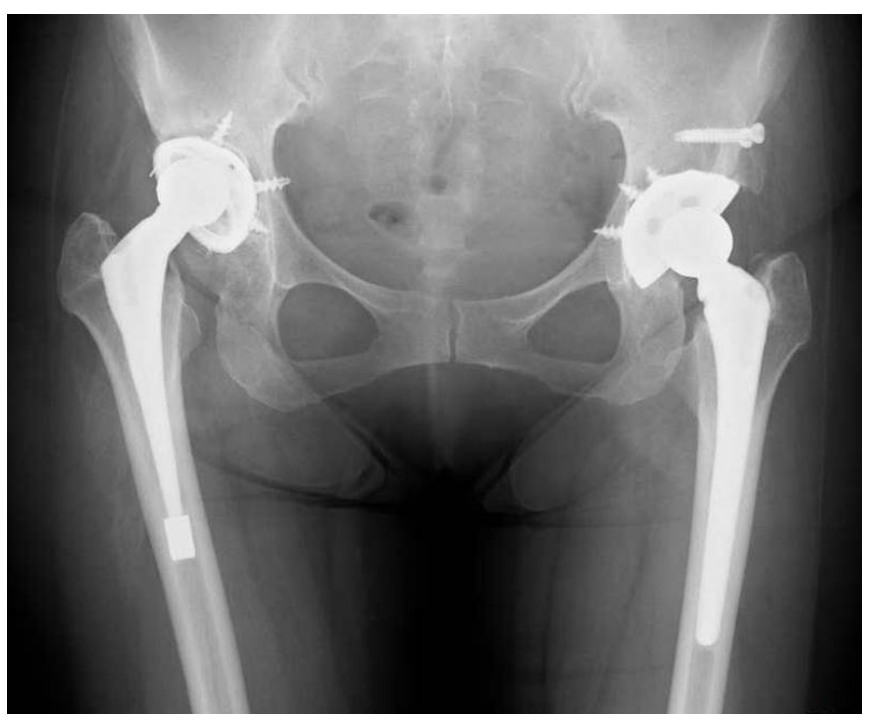

Fig. (1). A pre-revision pelvic radiograph. The right hip acetabular components, 16 years after the first THA, had loosened due to polyethylene wear. 
No clinical signs of infection were observed prior to the revision surgery.

The laboratory findings included a C-reactive protein (CRP) level of $0.06 \mathrm{mg} / \mathrm{dl}$ and a white blood cell (WBC) count of 6,600 cells $/ \mu 1$. The normal value for CRP in the hospital laboratory is a CRP level below $0.3 \mathrm{mg} / \mathrm{dl}$. During the revision surgery, after removing the ball, liner and shell, a cementless porous HA-coated acetabular component and polyethylene liner (AMS-HA acetabular shell and AMS liner; Kyocera, Kyoto, Japan) were implanted. In addition, a new metal ball (C-taper; Stryker, Mahwah, New Jersey, USA) was implanted (Fig. 2).

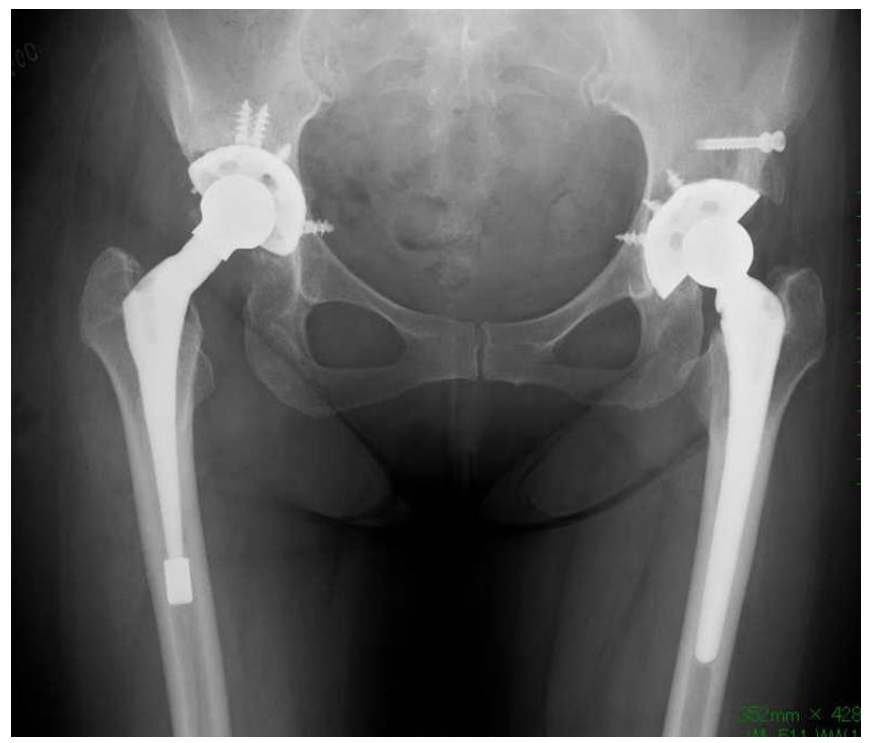

Fig. (2). A post-revision pelvic radiograph. The right hip acetabular components, shell, liner and ball were re-implanted.

With respect to the intraoperative findings, no signs of infection were observed and no specimens obtained intraoperatively for culture at the time of the revision surgery.

Following the revision surgery, the patient was given intravenous cefazolin at the time of induction of anesthesia and for 24 hours postoperatively. She exhibited a routine recovery and showed no symptoms of infection until 18 months after the revision surgery.

However, the patient reported gradually worsening right hip pain. Two weeks later, she visited our institution using a wheelchair. She was febrile $\left(38.7^{\circ} \mathrm{C}\right)$ and had a swollen right thigh with severe pain on motion of the hip joint; however, no fistulas were evidenced. The laboratory findings included a CRP level of $22.3 \mathrm{mg} / \mathrm{dl}$ and a WBC count of 5,500 cells $/ \mu 1$. The patient underwent aspiration of the right hip joint for culture; however, no aspirate was obtained.

Two-stage revision surgery was planned to treat the infected THA.

The patient underwent removal of the entire THA implant. There was pus in the capsule, and cup loosening was found. The patient underwent debridement of the area around the abscess in an effort to prevent progression of the infection. The area was thoroughly irrigated using pulsed lavage with 30 liters of saline. Finally, a cement mold was inserted into the femur (Fig. 3). The cement mold consisted of $120 \mathrm{~g}$ bone cement and $6 \mathrm{~g}$ of vancomycin. The patient was administered $1,200 \mathrm{mg}$ of linezolid for 14 days. Streptococcus mutans, a Gram-positive coccus was identified in all four of the intraoperative specimens (Table 1). The antibiotics were subsequently changed to a combination of $900 \mathrm{mg}$ of clindamycin and $1000 \mathrm{mg}$ of ampicillin.

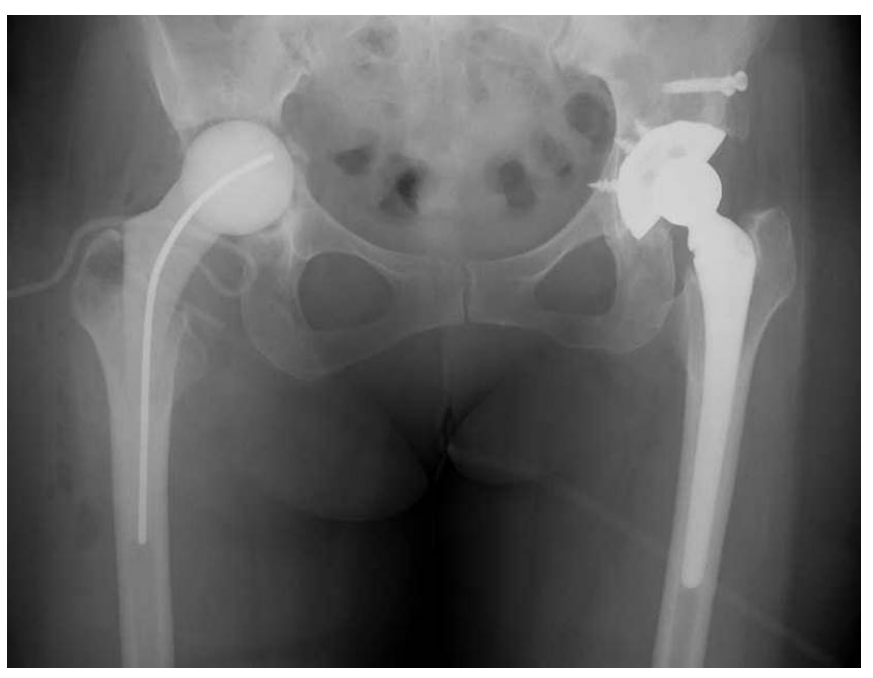

Fig. (3). A pelvic radiograph after the implant in the right hip was removed. The cement mold was implanted.

Table 1. Drug susceptibility.

\begin{tabular}{|c|c|c|c|c|c|}
\hline Drugs & MIC & Drugs & MIC & Drugs & MIC \\
\hline PCG & $\leq 0.06$ & ABPC & $\leq 0.25$ & CTM & $\leq 0.5$ \\
\hline CTRX & $\leq 0.5$ & CFPM & $\leq 0.5$ & CFDN & $\leq 0.25$ \\
\hline CDTR-PI & $\leq 0.25$ & PAPM/BP & $\leq 0.5$ & MEPM & $\leq 0.25$ \\
\hline GM & 4.0 & $\mathrm{AZM}$ & $\leq 0.5$ & CLDM & $\leq 0.25$ \\
\hline $\mathrm{VCM}$ & $\leq 1.0$ & LVFX & 2.0 & STFX & $\leq 0.12$ \\
\hline GRNX & $\leq 0.12$ & & & & \\
\hline \multicolumn{6}{|c|}{$\begin{array}{l}\text { CG: benzylpenicillin (penicillin G), ABPC: ampicillin, CTM: cefotiam, } \\
\text { TRX: ceftriaxone, CFPM: cefepime, CFDN: cefdinir, CDTR-PI: cefditoren pivoxil, } \\
\text { APM/BP: panipenem/betamipron, MEPM: meropenem, GM: gentamicin, } \\
\text { ZM: azithromycin, CLDM: clindamycin, VCM: vancomycin, LVFX: levofloxacin, } \\
\text { TFX: sitafloxacin (DU-6859), GRNX: garenoxacin Mesilate Hydrate (T-3811 ME). }\end{array}$} \\
\hline
\end{tabular}

The patient was referred to the department of dental surgery at our institution for an assessment of her dental problems. The dentist diagnosed her with caries.

However, Streptococcus parasanguinis, a resident of the flora of the oral cavity was identified only in specimens obtained from the patient's mouth. The laboratory findings related to infection were all within the normal ranges, and the patient underwent revision total hip arthroplasty eight weeks after the implant removal.

The operation was performed using an HA-coated cementless femoral component, an HA-coated cementless acetabular shell, a polyethylene liner and a zirconia ball (Kyocera, Kyoto, Japan).

A morselized allogenic bone graft was packed on the floor of the acetabulum (Fig. 4).

Following completion of the total revision surgery, the patient continuously received oral antibiotics consisting of 
$900 \mathrm{mg}$ of clindamycin and $1000 \mathrm{mg}$ of ampicillin for six months.

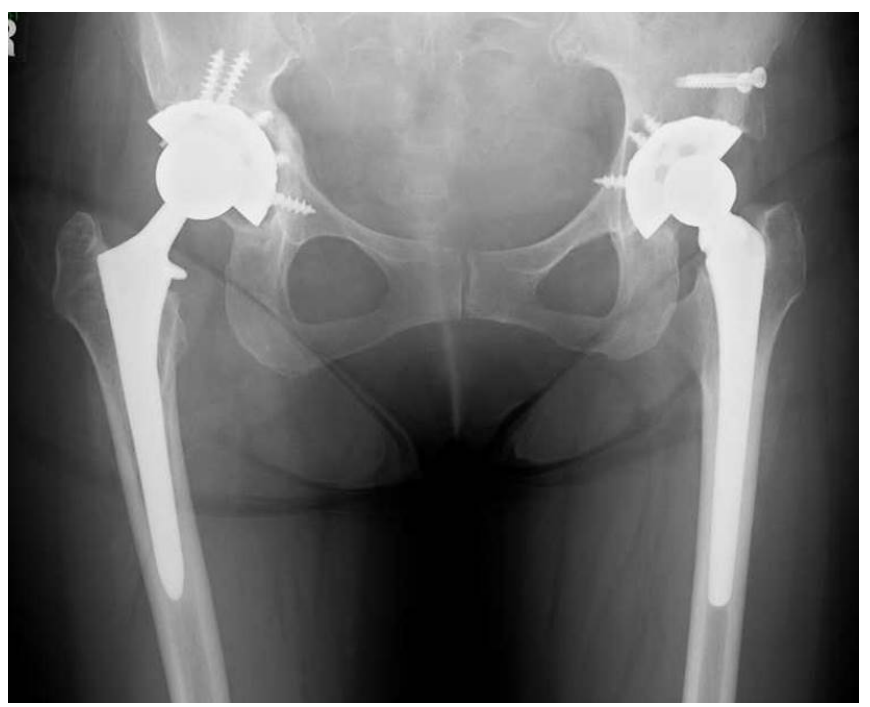

Fig. (4). A pelvic radiograph taken after the re-revision. The right hip components were implanted again.

The last follow-up occurred approximately 23 months after the total revision surgery, at which time, all laboratory findings indicative of infection remained well within the normal limits, no clinical signs of infection were noted and the prosthesis appeared to be well fixed radiographically. The patient is currently able to walk without a cane.

\section{DISCUSSION}

Infections of sites of total hip or knee arthroplasty due to hematogenous seeding of oral bacteria are very rare [11]. However, Maderazo et al. reported that oral bacteria are the cause of $15 \%$ of late infections of joint prostheses [3]. Several kinds of oral bacteria have been reported to be the causes of late infection following joint arthroplasty, many cases of which are due to Streptococcus viridans $[8,12]$. The Streptococcus mutans identified in the current case is a bacterium that causes dental caries; however, it is not a normal inhabitant of the mouth.

There are few reports of late infections of joint prostheses due to Streptococcus mutans [12]. In addition, many of the cases demonstrating late infections at the sites of joint prostheses due to oral bacteria occurred following such dental procedures as tooth extraction, periodontal scaling and endodontic surgery [8].

This is the first report of a late infection due to Streptococcus mutans following total hip arthroplasty in a patient without a history of dental procedures.

Although the use of antibiotic prophylaxis prior to dental procedures has been advocated in many reports in order to protect patients against periprosthetic joint infection [3-5, 13-15], this practice is controversial.

In contrast, Lockhart et al. [16] studied bacteremia in the context of tooth brushing and dental extraction and reported that simple tooth brushing provoked bacteremia in $32 \%$ of patients, which suggests that a typical patient is exposed to more than
200 episodes of bacteremia per year. In addition, Assael [17] reported that only a small proportion of cases of bacteremia originating from an oral source is associated with dental treatment and that it would not be reasonable to recommend that millions of patients be medicated at enormous cost simply based upon the findings of a few case reports.

There are several types of national guidelines/recommendations concerning the use of antibiotic prophylaxis before invasive dental procedures in patients undergoing joint replacement $[7,11]$. These recommendations are controversial. Some guidelines recommend appropriate antibiotic prophylaxis; however, they do not recommend the use of such prophylaxis in healthy patients with joint prostheses. The administration of antibiotic prophylaxis prior to dental treatment is recommended in patients with diabetes mellitus, rheumatoid arthritis, hemophilia or malignancy or those undergoing invasive dental treatment $[7,11]$.

In contrast, some guidelines do not recommend the use of antibiotic prophylaxis.

In 2003, The American Dental Association (ADA), in collaboration with the American Academy of Orthopedic Surgeons (AAOS) [2], issued an advisory statement recommending the use of antibiotic prophylaxis in patient with certain conditions, such as previous infection of an artificial joint, diabetes mellitus and others. However, in 2009, the AAOS $[7,11]$ stated that all patients with a history of total knee or hip arthroplasty are at an increased risk for bacteremia following dental procedures thus are considered to require antibiotic treatment prior to undergoing invasive dental procedures.

In addition, the most recent statements of the AAOS and ADA [18] issued in 2012 include the following three recommendations:

1. The practitioner may consider discontinuing the practice of routinely prescribing prophylactic antibiotics for patients with hip and knee prosthetic joint implants undergoing dental procedures.

2. We are unable to recommend for or against the use of topical oral antimicrobials in patients with prosthetic joint implants or other orthopedic implants undergoing dental procedures.

3. In the absence of reliable evidence linking poor oral health to prosthetic joint infection, it is the opinion of the work group that patients with prosthetic joint implants or other orthopedic implants maintain appropriate oral hygiene.

These recommendations reflect significant changes in the guidelines.

The current case suggests the possibility of hematogenous infection of joint prostheses in patients without a history of dental procedures and highlights the controversy surrounding the use of antibiotic prophylaxis prior to dental procedures. It is important to realize that the efficacy of antibiotic prophylaxis against joint infections has not yet convincingly been proven. Optimal dental hygiene and regular dental visits may be more important than antibiotic prophylaxis in maintaining joint health. Therefore, orthopedic surgeons should educate patients with joint prostheses about good oral health. 


\section{CONCLUSION}

Our report described a case of hematogenous infection of a hip prosthesis caused by oral bacteria. The incidence of such infections is not high; however, orthopedic surgeons are often asked to provide antibiotic prophylaxis for dental procedures by patients. Clinicians should reduce the use of unnecessary antibiotic prophylaxis. Treatments and procedures applicable to individual patients rely on mutual communication between the patient, physician, dentist and other healthcare practitioners. Realistically, orthopedic surgeons should prescribe antibiotic prophylaxis as appropriate after obtaining adequate informed consent.

\section{CONFLICT OF INTEREST}

The authors did not receive and will not receive any benefits or funding from any commercial party related directly or indirectly to the subject of this article.

\section{ACKNOWLEDGEMENTS}

Declared none.

\section{REFERENCES}

[1] American Dental Association, American Academy of Orthopaedic Surgeons, Advisory statement. Antibiotic prophylaxis for dental patients with total joint replacements. J Am Dent Assoc 1997; 128(7): 1004-8.

[2] American Dental Association, American Academy of Orthopedic Surgeons. Antibiotic prophylaxis for dental patients with total joint replacements. J Am Dent Assoc 2003; 134(7): 895-9.

[3] Maderazo EG, Judson S, Pasternak H. Late infections of total joint prostheses. A review and recommendations for prevention. Clin Orthop Relat Res 1988; 299: 131-42.

[4] Field E, Martin M. Prophylactic antibiotics for patients with artificial joints undergoing oral and dental surgery: necessary or not?. Br J Oral Maxillofac Surg 1991; 29(5): 341-6.
[5] Simmons N, Ball A, Cawson R. Case against antibiotic prophylaxis for dental treatment of patients with joint prostheses. Lancet 1992; 339: 301.

[6] MorrisAM, Howie S. Recommendations for antibiotics in patients with joint prosthesis are irresponsible and indefensible. J Can Dent Assoc 2009; 75(7): 513-5.

[7] Uçkay I, Pittet D, Bernard L, Lew D, Perrier A, Peter R. Antibiotic prophylaxis before invasive dental procedures in patients with arthroplasties of the hip and knee. J Bone Joint Surg Br 2008; 90(7): 833-8.

[8] LaPorte DM, Waldman BJ, Mont MA, Hungerford DS. Infections associated with dental procedures in total hip arthroplasty. J Bone Joint Surg Br 1999; 81(1): 56-9.

[9] Kaar TK, Bogoch ER, Devlin HR. Acute metastatic infection of a revision total hip arthroplasty with oral bacteria after noninvasive dental treatment. J Arthroplasty 2000; 15(5): 675-8.

[10] Brown ML, Drinkwater CJ. Hematogenous infection of total hip arthroplasty with actinomyces following a noninvasive dental procedure. Orthopedics 2012; 35(7): e1086-9.

[11] Olsen I, Snorrason F, Lingaas E. Should patients with hip joint prosthesis receive antibiotic prophylaxis before dental treatment? J Oral Microbiol 2010; 30: 2.

[12] Waldman BJ, Mont MA, Hungerford DS. Total knee arthroplasty infections associated with dental procedures. Clin Orthop Relat Res 1997; 343: 164-72

[13] Rubin R, Salvati E, Lewis R. Infected total hip replacement after dental procedures. Oral Surg Oral Med Oral Pathol 1976; 41(1): 18-23.

[14] Downes E. Late infection after total hip replacement. J Bone Joint Surg Br 1977; 59(1): 42-4.

[15] Stinchfield FE, Bigliani LU, Neu HC, Goss TP, Foster CR. Late haematogenous infection of total joint replacement. J Bone Joint Surg Am 1980; 62(8): 1345-50.

[16] Lockhart PB, Brennan MT, Sasser HC, Fox PC, Paster BJ, Bahrani-Mougeot FK. Bacteremia associated with tooth brushing and dental extraction. Circulation 2008; 117(24): 3118-25.

[17] Assael LA. Oral bacteremia as a cause of prosthesis failure in patients with joint replacements. J Oral Maxillofac Surg 2009; 67(9): 1789-90.

[18] American Academy of Orthopedic Surgeons, American Dental Association, "Prevention of Orthopedic Implant Infection in Patients Undergoing Dental Procedure. Evidence-Based Guideline and Evidence Report. AAOS Clinical Practice Guideline 2012; Unit v0.2 2.2". Available from: http://www.aaos.org/research/ guidelines/PUDP/PUDP_guid eline.pdf 